\title{
Wheel-running and rest activity pattern interaction in two octodontids (Octodon degus, Octodon bridgesi)
}

\author{
ADRIÁN OCAMPO-GARCÉS ${ }^{1,2,3}$, FELIPE HERNÁNDEZ ${ }^{3}$, WILSON MENA ${ }^{1}$ and \\ ADRIÁN G PALACIOS ${ }^{1}$
}

\author{
${ }^{1}$ Centro de Neurociencias de Valparaíso, Universidad de Valparaíso \\ 2 Departamento de Neurología y Neurocirugía, Hospital Clínico de la Universidad de Chile \\ ${ }^{3}$ Programa de Fisiología y Biofísica, Instituto de Ciencias Biomédicas, Facultad de Medicina, Universidad de \\ Chile
}

\begin{abstract}
Wheel-running and other non-photic stimuli influence the rest-activity pattern of diurnal and nocturnal mammals. A day to night inversion of phase preference of activity was described among Octodon degus, when exposed to ad-libitum wheel running. We have studied the rest-activity pattern response in presence of ad libitum wheel-running in wild-captured male individuals from two species of genus Octodon: O. degus (n=9, crepuscular-diurnal) and $O$. bridgesi $(\mathrm{n}=3$, nocturnal). After two months of habituation to laboratory conditions, recordings were performed in isolation chambers under a 12:12 light-dark schedule with or without access to a running wheel. Actograms were constructed from data obtained by an automated acquisition system. $O$. bridges $i$ were also recorded under constant darkness, with or without access to wheelrunning. Entrained to the light-dark schedule, a crepuscular pattern of activity was evident for $O$. degus, whereas $O$. bridgesi displayed a robust nocturnal chronotype. The activity of $O$. degus observed during the dark phase was enhanced when wheel-running was allowed. No significant change in phase preference was observed for $O$. bridgesi when wheel-running was allowed. A lengthening of endogenous period was observed in $O$. bridgesi after wheel-running exposure under constant darkness. Nocturnal and diurnal octodontids exhibit different masking responses to wheel-running.
\end{abstract}

Key terms: circadian rhythms, masking, Octodon degus, Octodon bridgesi, wheel-running

\section{INTRODUCTION}

The circadian system of mammals possesses a central pacemaker (the hypothalamic suprachiasmatic nuclei) that oscillates with a period close to 24 hours. This rhythm is transferred downstream to multiple effectors of the circadian system (van Esseveldt et al., 2000). The overt rhythms displayed by circadian effectors determine the species-specific temporal niche. Growing evidence suggests that diurnal and nocturnal mammals share common properties at the central oscillator level (Smale et al., 2003), and it has been proposed that neural processes that determine differences between diurnal and nocturnal mammals exist downstream of the suprachiasmatic nuclei (Schwartz et al., 2004).

Non-photic stimuli such as wheelrunning influence the rest-activity pattern of diurnal and nocturnal mammals. A diurnal to nocturnal inversion of activity phase preference has been described in captive-bred Octodon degus exposed to adlibitum wheel-running (Kas and Edgar, 1999). The enhancement of nocturnal activity occurs almost immediately after the exposure to the non-photic stimulus. The absence of a transient in the phase adjustment suggests that the critical process is not related to changes in the phase or period length of the central circadian

Corresponding author: Adrián Ocampo-Garcés MD PhD, Programa de Fisiología y Biofísica, Facultad de Medicina, Universidad de Chile, Independencia 1027, Casilla 70005, Correo 7, Santiago, Chile, Phone: (56-2) 678-6423, Fax: (56-2) 777-6916,email: aocampo@med.uchile.cl 
oscillator. In the crepuscular-diurnal murid Arvicanthis niloticus (nile grass rat), an enhancement of activity is observed during dark phase under wheel-running exposure (Blanchong et al., 1999). Recent evidence suggests that a masking process (direct action on effectors downstream of the circadian oscillator) appears to be the cause of this phenomenon among A. niloticus (Redlin and Mrosovsky, 2004). Field results demonstrated that spatial and temporal shifts in the activity of degus involves a tradeoff based on avoidance of exposure to heat and the search for food (Bacigalupe et al., 2003). The homeostatic strategy of avoiding heat exposure by favoring activity during the dark phase in crepuscular diurnal rodents may be mediated by a non-photic masking mechanism targeting effectors of the circadian system (Kas and Edgar, 1999).

The octodontidae family (Rodentia) has been observed to include both nocturnal and crepuscular species, particularly among species of the monophyletic genus Octodon (Honeycutt et al., 2003). A crepusculardiurnal pattern of activity has been described for $O$. degus in wild (Kenagy et al., 2002) and captive-bred populations (Labyak et al., 1997; Kas and Edgar, 1998; García-Allegue et al., 1999; Hernández et al., 2003). O. bidgesi exhibits a nocturnal phase preference in the field as estimated by the timing of captures (Muñoz and Murúa, 1987; Verzi and Alcover, 1990). A unimodal, midnight-centered phase of activity has been observed under controlled conditions for $O$. bridgesi (Ocampo-Garcés and Palacios, 2001) and O. lunatus (Ocampo-Garcés et al., 2003). Here we explore the masking response of the restactivity cycle to ad-libitum wheel-running in wild-captured specimens of the diurnalcrepuscular $O$. degus and the nocturnal $O$. bridgesi.

\section{METHODS}

All individuals recorded were adult male individuals captured in their natural environment. Nine degus (190-230 g.) and three $O$. bridgesi (180-210 g.) were captured by means of baited medium-sized
Sherman traps. Animals were maintained under a 12:12 Light-Dark (LD) schedule for a two-month period of adaptation to captivity conditions. A wheel placed inside the maintenance cage allowed the animals to become familiarization with wheelrunning.

One week prior to the recording session, the animals were installed in recording cages $(30 \times 40 \times 30 \mathrm{~cm})$ contained in individual isolation chambers $(65 \times 60 \times 60$ $\mathrm{cm}$ ) and maintained under a 12:12 LD schedule. Light intensity was set at 300 luxes at the ground level during light hours. Ambient temperature was maintained at 21$23^{\circ} \mathrm{C}$, with food and water ad libitum. A blocked running-wheel was inside the recording cage.

\section{Protocol}

Entrained condition ( $O$. degus and $O$. bridgesi): Time was expressed as Zeitgeber Time (ZT), with lights-on at ZT 00 . Recording started with at least ten days of entrained period (12:12 LD schedule) with running wheel blocked (LD-NO-WHEEL condition). The running wheel was left unblocked for at least one week (LDWHEEL condition) thereafter, starting at lights-off (ZT 12) of the first LD-WHEEL condition day. Following this period, a second LD-NO-WHEEL condition was recorded. After the second period under the LD-NO-WHEEL condition, O. bridgesi individuals were maintained under constant darkness to obtain a free-running record for at least three-weeks without access to the running wheel (DD-NO-WHEEL condition). Finally, the wheel inside recording chamber was unblocked for at least three weeks under constant darkness (DD-WHEEL condition).

\section{Data acquisition}

An automated computer-based acquisition system designed for long term sleep-wake recording was adapted to record rest activity rhythm (Vivaldi et al., 1994). The system recorded and stored the activity counts detected by a piezoelectric device placed at the base of the animal's cage 
originally in 15-second intervals. Activity counts were later collapsed into 10-minute intervals (144 per day).

\section{Data analysis and statistics}

To describe the rest-activity pattern under the entrained condition (LD-NO-WHEEL and LD-WHEEL), light or dark phase predilection of activity and fraction of activity counts occurring during the dark phase were obtained. Activity phase predilection was assessed individually by pairing activity counts obtained during light and dark phases during recording days. Predilection was defined as whether the animal displayed a significant difference in activity counts between light or dark phases during recording days (two-tailed paired $t$ test). Individual values for fraction of activity counts occurring during the dark phase (expressed as percentages) were estimated from the mean activity curve obtained during LD-WHEEL and LD-NOWHEEL days. To assess the effect of wheel-running on the rest-activity pattern under entrained condition, a WHEEL $v s$. NO-WHEEL two-tailed paired $t$-test was applied to the fraction of activity during dark phase obtained in pool of animals of each species. Raster plots of $O$. bridgesi activity were double plotted to visually assess the effect of wheel-running on the free-running period under constant darkness.

\section{RESULTS}

The effect of wheel-running on the rest activity pattern in a representative $O$. degus is presented on Figure 1. An almost immediate response could be seen in the activity phase preference that drifted to nocturnal phase. This response was reversed after the wheel was removed. On the contrary, the rest activity pattern of $O$. bridgesi appeared to be unaffected during ad-libitum wheel running exposure.

On average ( \pm S.E.M) O. degus individuals concentrated $50.0 \pm 6.4 \%$ of their activity counts during the light phase under LD-NO-WHEEL condition (Fig. 2).
During ad libitum wheel-running exposure (LD-WHEEL condition), the fraction of nocturnal activity counts increased to $60.5 \pm 6.2 \% \quad(\mathrm{p}<0.05$, two-tailed paired $t$-test). A crepuscular bimodal pattern with a predominant bout of activity at dusk is preserved under both experimental conditions.

$O$. bridgesi specimens displayed a nocturnal predominance of active phase under LD-NO-WHEEL condition, with $92.5 \pm 1.5 \%$ of activity counts occurring during dark phase. Under LD-WHEEL condition, $84.2 \pm 5.3 \%$ of daily activity counts occurred at night. The locomotor activity profile always exhibits an immediate and transient peak after lightsoff and a decrement preceding lights-on. In two of the $O$. bridgesi specimens, the active phase exhibited a midnight-centered unimodal profile under entrained conditions. The nocturnal activity preference remained almost unaffected under the LD-WHEEL condition. The restactivity pattern of a representative $O$. bridgesi under four experimental conditions is double-plotted in Figure 3. After unblocking the wheel inside the cage (DDWHEEL condition), the free running period gradually adopted a value greater than 24 hours. The free-running period lengthened in the three individuals recorded under DDWHEEL condition.

\section{DISCUSSION}

Non-photic stimuli, such as wheel-running (Hut et al., 1999), feeding (Castillo et al 2004), or social activity (Goel and Lee, 1995), could affect the period and/or phase of the central circadian oscillator in rodents. A Phase Response Curves (PRC) and Period (tau) Response Curves has been described for a number of non-photic stimuli (Yanielli and Harrington, 2004). Phase advances primarily occur during the subjective day, and phase delays have been observed during the subjective night. Advances and delays caused by scheduled wheel-running (Hut et al., 1999) or feeding (Castillo et al., 2004) could be strong enough to entrain the rest-activity rhythm to 


\section{Octodon degus}
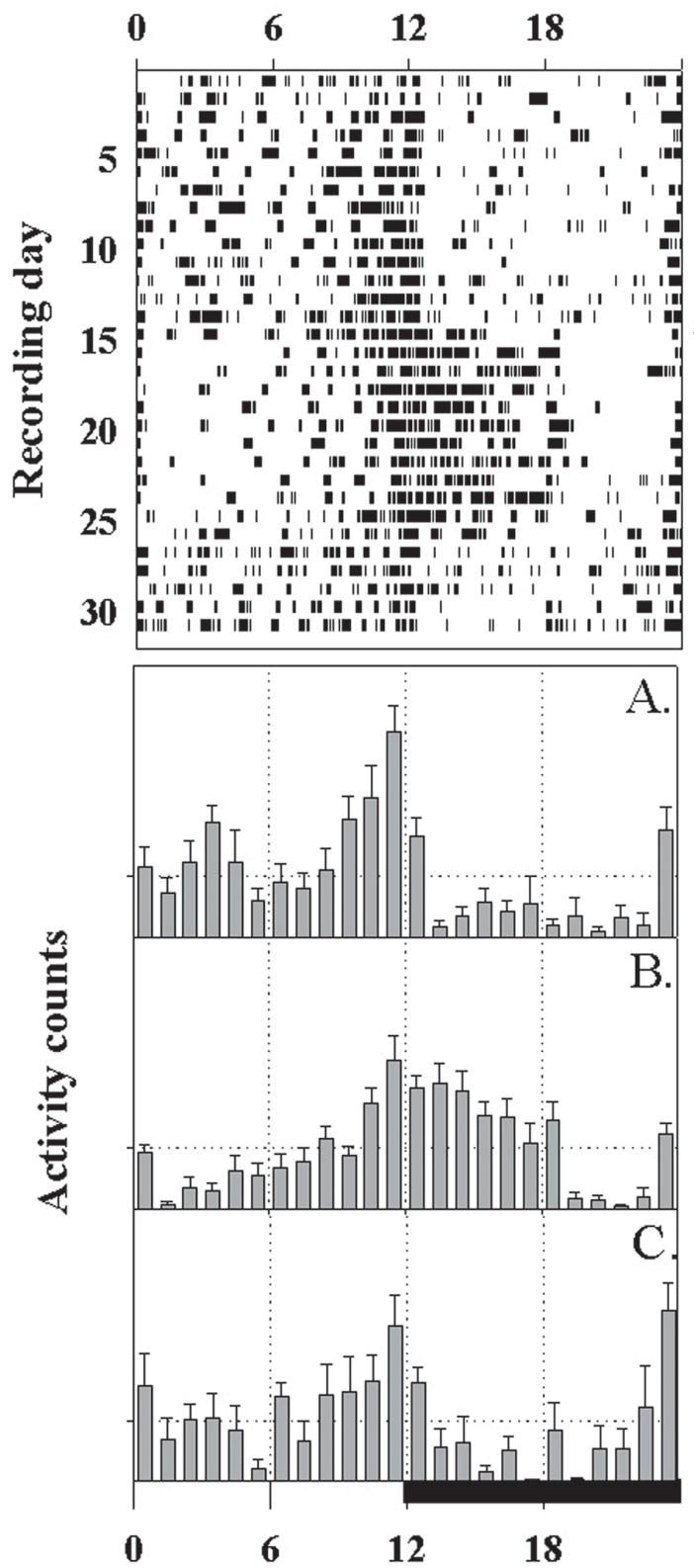

Zeitgeber time
Octodon bridgesi
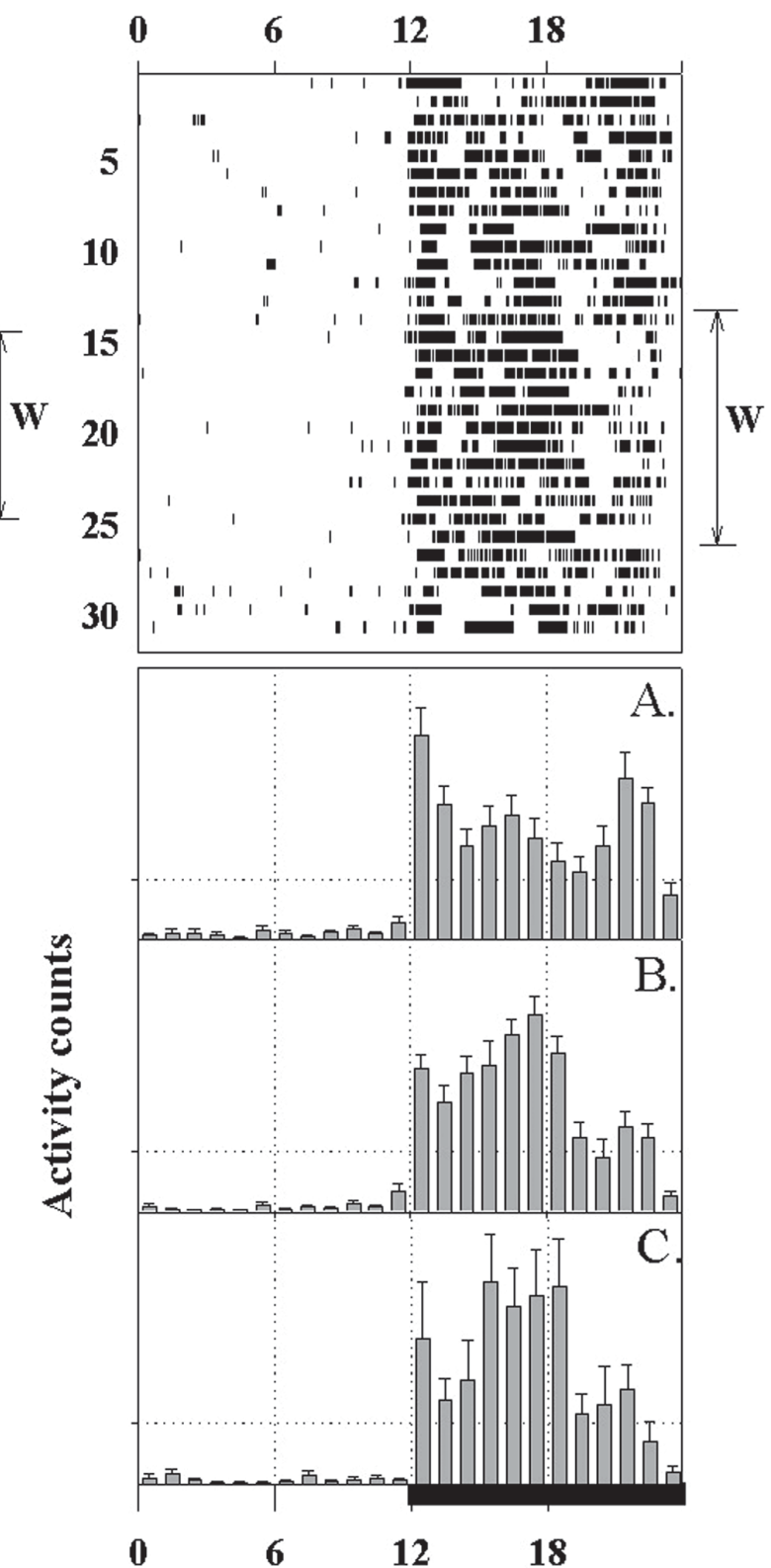

Zeitgeber time

Figure 1. Rest-activity records obtained under a 12:12 light-dark schedule. One-minute resolution raster plot (top), and mean activity curve (hourly average \pm S.E.M) obtained during the corresponding recording period (lower panel) of representative Octodon degus (left) and $O$. bridgesi (right). Vertical marks in raster plots correspond to minutes pertaining to the highest quintile of activity of the corresponding day. Ad libitum wheel-running period (W) is indicated at the right axis. In the lower panel, A summarizes LD-NO-WHEEL condition, B corresponds to LDWHEEL condition, and $\mathrm{C}$ to the period following the return to the LD-NO-WHEEL condition. Activity counts are expressed as a percentage of daily mean (horizontal dotted line). Time is represented in hours after lights-on (Zeitgeber time). The dark phase is indicated by a horizontal filled bar at the abscissa. 
Octodon degus

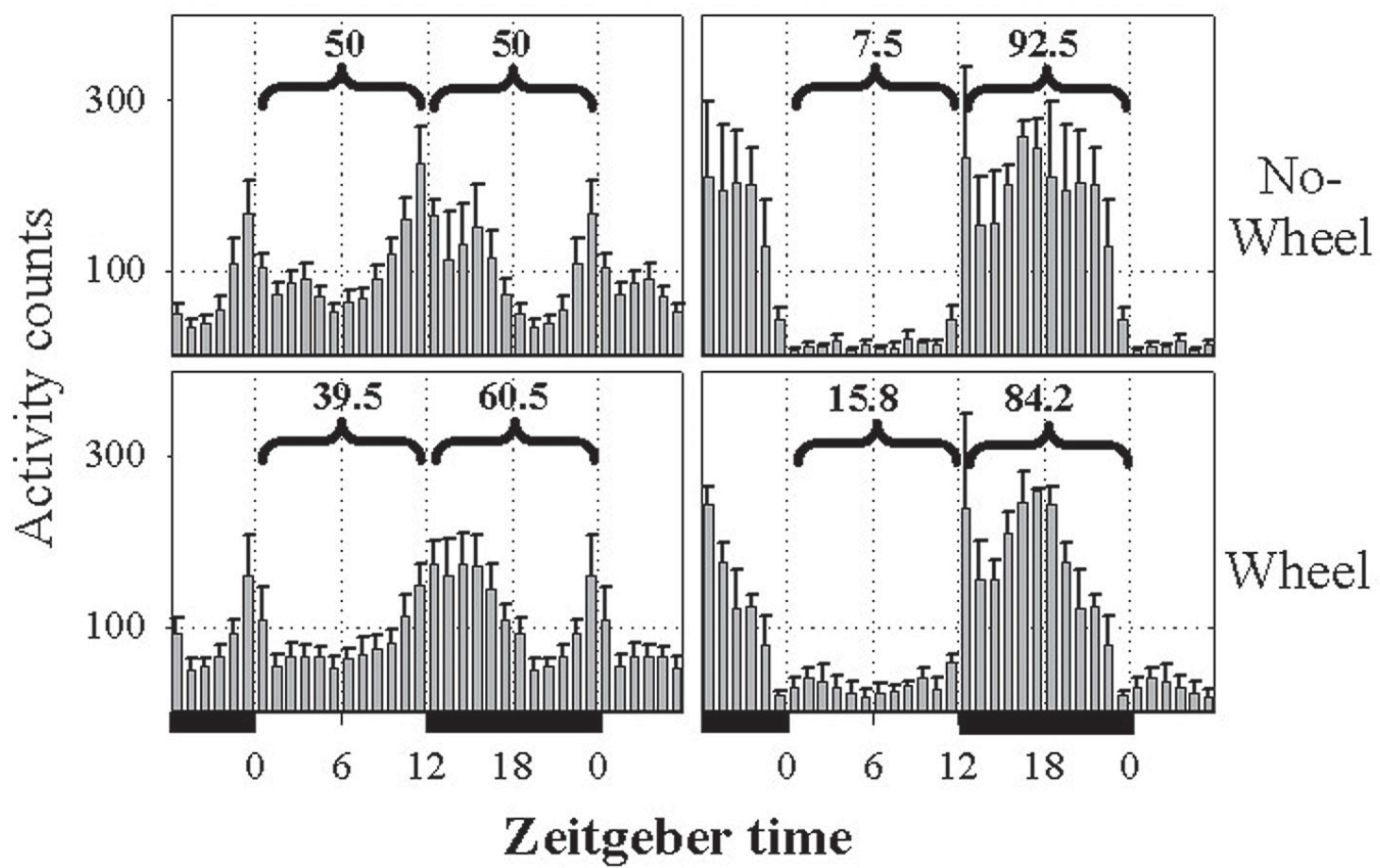

Figure 2. Mean activity curve (hourly average \pm S.E.M) obtained under LD-WHEEL and LD-NOWHEEL conditions of $O$. degus $(\mathrm{n}=9)$ and $O$. bridgesi $(\mathrm{n}=3)$. Activity counts are expressed as a percentage of daily mean activity (horizontal dotted line). Values on the top of each graphic correspond to the fraction of diurnal and nocturnal activity (in percentage). The dark phase is indicated by a horizontal filled bar at the abscissa. ZT18-23 and ZT 00-05 intervals are double plotted.

non-photic Zeitgebers scheduled at periods close to 24 hours. Based on scheduled wheel-running experiments, a PRC has been inferred for the diurnal sciurid (Rodentia) Spermophylus citellus that coincide with that observed among nocturnal rodents (Hut et al., 1999). In our results, a nocturnal preference of activity was evident among $O$. bridgesi individuals entrained to a 12:12 light-dark schedule (Fig. 2). The active phase started to free run at the expected scotopic-phase, and thus the active phase occurred during the subjective night (Fig 3). Previous reports have demonstrated that the free-running period of $O$. degus is dramatically lengthened when scheduled wheel-running fell at the end of the subjective night (Kas, 1999). The observed lengthening of the free-running period during DD-WHEEL condition in $O$. bridgesi could be explained by a non-photic phase delay or lengthening of the period of the rest-activity rhythm in response to wheel-running during the subjective night. These results suggest that wheel running occurring during the subjective night exerts a similar response at the central oscillator on diurnal and nocturnal Octodon species. 


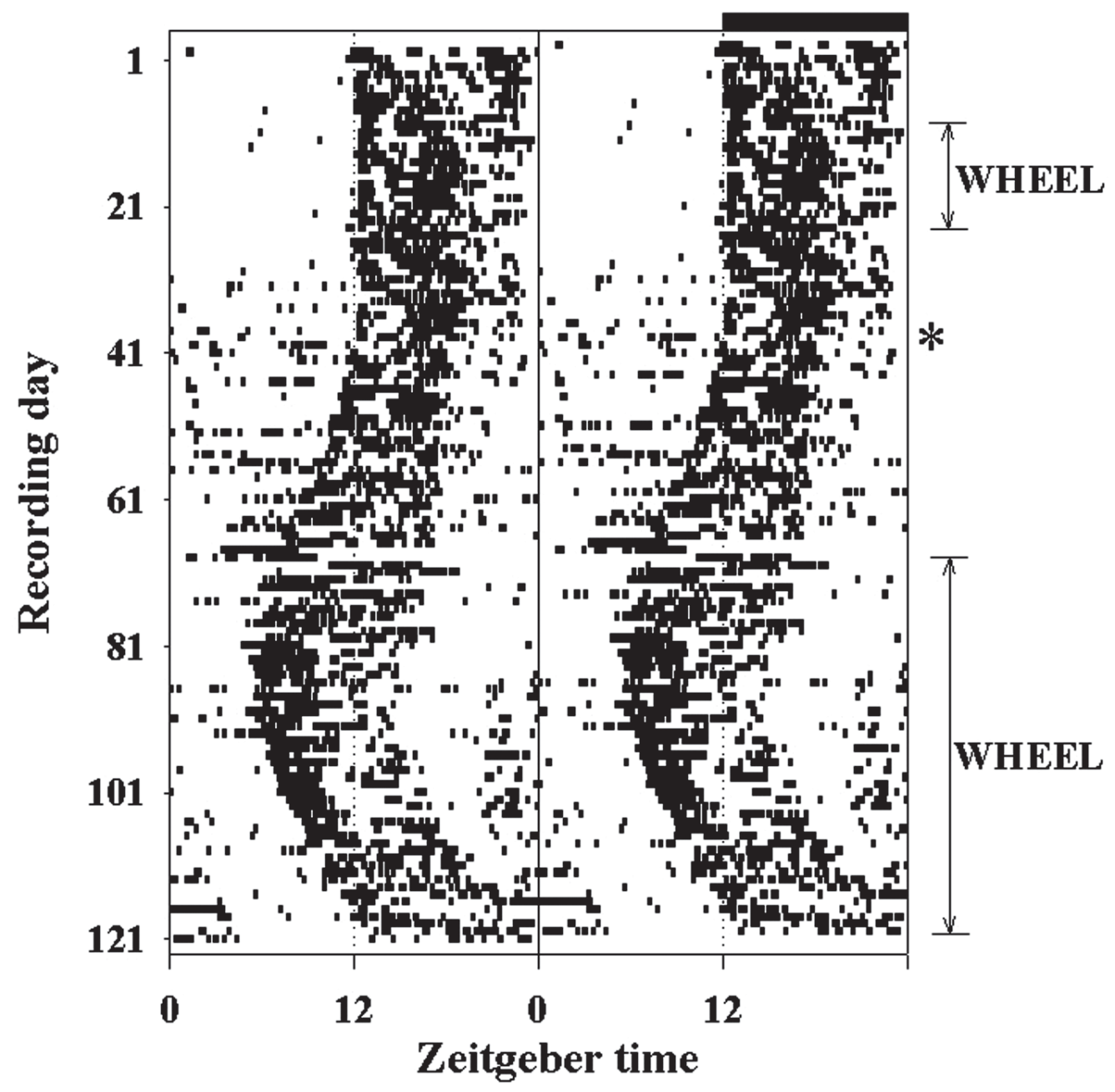

Figure 3. Double plotted actograms obtained under entrained (LD) and free running condition (DD) in a representative cases of $O$. bridgesi. Vertical marks in raster plots correspond to minutes pertaining to the highest quintile of activity of the corresponding day. The ad libitum wheel-running period is indicated at the right axis. The beginning of free-running is indicated by an asterisk. The dark phase at the initial conditions is indicated by a horizontal filled bar at the upper abscissa.

Entrained to a 12:12 light-dark cycle, adlibitum non-photic stimulus such as wheelrunning could also play an important role in sculpturing rest-activity profiles by modifying the phase preference of activity, as has been observed for O. degus (Kas and Edgar, 1999) and A. niloticus (Banchong et al., 1999). Our results in wild-captured $O$. degus are consistent with the aforementioned data. Entrained to a 12:12 light dark schedule, an almost instantaneous and reversible drift to a nocturnal preference of activity was observed among $O$. degus (Figs. 1 and 2). In contrast, the nocturnal $O$. bridgesi did not display a significant change in the phase preference of activity under entrained conditions (Fig. 2). Kas and Edgar (1999) proposed that a non-photicresponsive element operates as a target of an incoming feed-back signal from yet unknown effectors of the circadian system. This non-photic-responsive element is believed to operate as an interface that switches the output signal of the main circadian oscillator determining phase preference of activity. In the case of the crepuscular-diurnal murid A. niloticus, when subjected to a short light-dark cycle, an 
enhancement of wheel running occurs during the subjective day and subjective night, whenever a scheduled short dark interval took place (Redlin and Mrosovsky, 2004). This result is consistent with a masking process that interferes with the output signal of the central oscillator of the circadian system.

In sum, we found that two closely related species that exhibit different temporal niches display divergent responses to a non-photic masking stimulus. The masking of the rest activity pattern under entrained conditions induced by wheel-running is only observed among the crepuscular-diurnal O.degus. The lack of masking response of the rest-activity pattern in $O$. bridgesi suggests that some non-photic responsive elements are absent in this nocturnal rodent. Our results coincide with the hypothesis that the evolutionary drift to a more diurnal phase preference is associated with the emergence of regulatory interfaces downstream of the circadian oscillator.

\section{ACKNOWLEDGMENTS}

Thanks are due to Drs. Rodrigo Vásquez and Bárbara Saavedra in providing wildcaptured $O$. bridgesi specimens; Dr. Claudia Cecchi, who helped us in capturing wild $O$. degus specimens; Mr. Juan Peirano and Dr. Ennio Vivaldi for providing data acquisition methodologies; and Mr. Cristián López for excellent animal care. This research was supported by FONDECYT Grant 3010028.

\section{REFERENCES}

BACIGALUPE LD, REZENDE EL, KENAGY GJ, BOZINOVIC F (2003) Activity and space use by degus: A trade-off between thermal conditions and food availability? J Mammal 84: 311-318

BLANCHONG JA, MCELHINNY TL, MAHONEY MM, SMALE L (1999) Nocturnal and diurnal rhythms in the unstriped nile rat, Arvicanthis niloticus. J Biol Rhythms 14:364-377

CASTILLO MR, HOCHSTETLER KJ, TAVERNIER JR RJ, GREENE DM, BULT-ITO A (2004) Entrainment of the master circadian clock by scheduled feeding. Am J Physiol 287: R551-R555

GARCÍA-ALLEGUE R, LAX P, MADARIAGA AM, MADRID, JA (1999) Locomotor and feeding activity rhythms in a light-entrained diurnal rodent, Octododon degus. Am J Physiol 277: R523-531

GOEL N, LEE TM (1995) Sex differences and effects of social cues on daily rhythms following phase advances in Octodon degus. Physiol Behav 58:205-213

HERNÁNDEZ F, OCAMPO-GARCÉS A, VIVALDI EA (2003) REM sleep in Octodon degus under entrained conditions. Biol Res 36: R96

HONEYCUTT RL, ROWE DL, GALLARDO MH (2003) Molecular systematics of the South American caviomorph rodents: relationships among species and genera in the family Octodontidae. Mol Phylogenet Evol 26: 476-489

HUT RA, MROSOVSKY N, DAAN S (1999) Nonphotic entrainment in a diurnal mammal, the european ground squirrel (Spermophilus citellus). J Biol Rhythms 14: 409-419

KAS MJ (1999) Sleep and circadian timekeeping in Octodon degus. Thesis. The Netherlands: Groningen University

KAS MJ, EDGAR D (1998) Crepuscular rhythms of EEG sleep-wake in a hystricomorph rodent, Octodon degus. J Biol Rhythms, 13: 9-17

KAS MJ, EDGAR D (1999) A nonphotic stimulus inverts the diurnal-nocturnal phase preference in Octodon degus. J Neurosci, 19:328-33

KENAGY GJ, NESPOLO RF, VÁSQUEZ RA, BOZINOVIC F (2002) Daily and seasonal limits of time and temperature to activity of degus. Rev Chil Hist Nat 75: 567-581

LABYAK SE, LEE TM, GOEL N (1997) Rhythm chronotypes in a diurnal rodent, Octodon degus. Am J Physiol 273: R1058-R1066

MUÑOZ A, MURÚA R (1987) Biología de Octodon bridgesi bridgesi (Rodentia, Octodontidae) en la zona costera de Chile central. Boletín de la Sociedad de Biología de Concepción (Chile) 58: 107-117

OCAMPO-GARCÉS A, PALACIOS AG (2001) Chronobiology of rest activity rhythm of Octodon bridgesi in captivity. Biol Res 34: R-41

OCAMPO-GARCÉS A, VALLADARES JP, PALACIOS AG (2003) Octodon lunatus: A nocturnal octodontid of northern Chile. Sleep 26: A117

REDLIN U, MROSOVSKY N (2004) Nocturnal activity in a diurnal rodent (Arvicanthis niloticus): the importance of masking. J Biol Rhythms 19: 58-67

SCHWARTZ MD, NUNEZ AA, SMALE L (2004) Differences in the suprachiasmatic nucleus and lower subparaventricular zone of diurnal and nocturnal rodents. Neuroscience 127: 13-23

SMALE L, LEE T, NUNEZ AA (2003) Mammalian diurnality: some facts and gaps. J Biol Rhythms 18: 356-366

VAN ESSEVELDT LE, LEHMAN MN, BOER GJ (2000) The suprachiasmatic nucleus and the circadian timekeeping system revisited Brain Res Rev 33: 34-77

VERZI DH, ALCOVER A (1990) Octodon bridges, Waterhouse 1844 (Rodentia: Octodontidae) in the Argentinian living mammalian fauna. Mammalia, 54:61-67

VIVALDI EA, WYNEKEN U, RONCAGLIOLO M, OCAMPO A, ZAPATA A (1994) Measures of location and dispersion of sleep state distributions within the circular frame of a 12:12 light:dark schedule in the rat. Sleep, 17:208-219

YANNIELLI P, HARRINGTON ME (2004) Let there be "more" light: enhancement of light actions on the circadian system through non-photic pathways. Prog Neurobiol 74:59-76 